\title{
Office-Based Laser Surgery for Benign Laryngeal Lesion
}

\author{
Soo Min Hwang \\ Doh Young Lee \\ Nu-Ri Im \\ Hyun-Ji Lee \\ Byoungjae Kim \\ Kwang-Yoon Jung \\ Tae Hoon Kim \\ Seung-Kuk Baek
}

Department of Otorhinolaryngology-Head and Neck Surgery, Korea University College of Medicine, Seoul, Korea
Received November 28, 2015

Revised December 8, 2015

Accepted December 9, 2015

\section{Background and Objectives}

The aim of this clinically feasible study was to show the preliminary result regarding postoperative outcomes of in-office laser surgery for a benign laryngeal lesion.

\section{Materials and Methods}

This clinically feasible trial included 12 patients suffering from various laryngeal pathology. Vocal fold polyp was most common ( $n=3)$, followed by granuloma $(n=2)$, papillomatosis ( $n=2)$, and glottal leukoplakia/dysplasia $(n=2)$. Laser vaporization was performed using the diode laser set at $12 \mathrm{~W}$ output power and a fiberoptic laryngoscope.

\section{Results}

None of the patients showed complication. Three cases of vocal polyp were cured by a single procedure. In cases of leukoplakia and laryngeal hair, the lesion was significantly improved but required repeated procedures. In contrast, patients with contact granuloma, subglottic stenosis, and tracheal lesion showed partial remission with laser surgery. Only one patient required conversion to general anesthesia after office-laser surgery; this patient had laryngeal papillomatosis with an invisible surface and inappropriate exposure, making it difficult for the fiberoptic endoscope to reach the lesion.

\section{Conclusion}

Efficacy of in-office laser surgery for benign laryngeal disease is comparable to that of conventional laryngeal microsurgery under general anesthesia. Further comparative study is necessary to elucidate the long-term results.

\section{Key words}

Diode laser; Local anesthesia; Fiberoptic; Office-based

\footnotetext{
Correspondence

Seung-Kuk Baek

Department of Otorhinolaryngology-Head and Neck Surgery, Korea Univsersity Anam Hospital, 73, Inchon-ro, Seongbuk-gu, Seoul 02841, Korea Tel: $+82-2-920-6618$

Fax: $+82-2-920-5233$

E-mail: mdskbaek@agmail.com

(C) Korean Society for Laser Medicine and Surgery

(c) This is an open access article distributed under the terms of the Creative Commons Attribution NonCommercial License (http://creativecommons.org/ licenses/by-nc/4.0) which permits unrestricted noncommercial use, distribution, and reproduction in any medium, provided the original work is properly cited.
} 


\section{INTRODUCTION}

Treatment of benign lesion of larynx is often bothersome due to necessity of general anesthesia for accurate procedure. Specifically, superficial epithelial disease of the vocal folds require delicate handling of the lesion to improve the postoperative voice quality. ${ }^{1}$ Office-based laryngeal surgery was performed since the nineteenth century. With development of direct-laryngoscopic guided laryngeal surgery optimized by assistants administering anesthesia and procedural support, relocation of the procedure to the operating room began to occur in early twentieth century. ${ }^{2}$ In the past, cases of recurrent laryngeal papillomas, glottal leukoplakia, and either laryngeal or tracheal stenosis often required multiple surgical procedures performed under general anesthesia. However, many of aforementioned conditions can now be effectively and safely treated in the office through laser surgery that utilizes only the topical anesthesia to minimize the operation time and morbidity from general anesthesia. ${ }^{3-5}$ Recent advancements in distal-chip flexible endoscopic technology and fiber-based lasers have enabled such office-based laryngeal laser surgery. In this study, we aimed to evaluate the feasibility of office-based laser surgery for benign laryngeal lesion.

\section{MATERIALS AND METHODS}

\section{Patients}

We prospectively included 12 patients with various benign laryngeal pathologies from July 2013 to July 2014. Vocal fold polyp was most common ( $n=3)$, followed by granuloma ( $n=2)$, papillomatosis ( $n=2)$, glottal leukopla$\mathrm{kia} /$ dysplasia (n=2). Most of the cases did not receive any previous treatment, except 1 patient with leukoplakia who underwent laryngeal microsurgery for surgical biopsy under general anesthesia. All patients refused surgical procedure under general anesthesia due to either simple refusal or inappropriate general condition for general anesthesia.

\section{Procedure}

The procedure was performed under local anesthesia in the outpatient clinic. Patients are placed in sitting position and both topical lidocaine and epinephrine spray are applied to nasal cavity to minimize pain as the fiberoptic endoscope enters. $2 \%$ lidocaine was injected into the larynx and proximal trachea through the port on the endoscope in order to anesthetize lower pharynx and larynx. D15 diode laser fiber (Ceralas, Bonn, Germany) is inserted through the working channel ( $3.7 \mathrm{~mm}$ in diameter) of the video fiberoptic esophagoscope (EE-1580K, PENTEX, NJ) (Fig. 1). The laser power is set at 12 Watt and is fired in continuous superpulse mode. Vaporization technique is used to remove the lesion, where laser is fired once the laser tip is placed onto the lesion. All staffs wore protective eyewear, and the surgeon observed the surgical field through the monitor. The fiber enters the portal of the laryngoscope channel that is held by an assistant. Vital status of all patients were monitored during the surgery, as well as 30 minutes after the surgery in the waiting room. Pain, dysphagia, and dyspnea with 02 saturation and pulse were checked. If needed, patients were placed on 7 days of voice rest, 3 weeks of voice conservation, and scheduled for a follow-up appointment 4 weeks after the surgery.

\section{RESULTS}

Table 1 summarizes the results. There was no postprocedural complications. Three cases of vocal polyp were cured by single procedure (Fig. 2). In cases of leu-

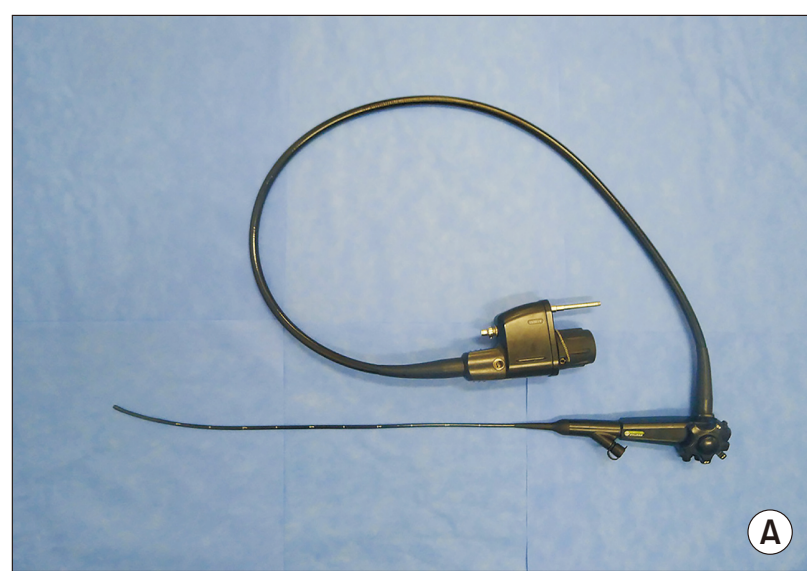

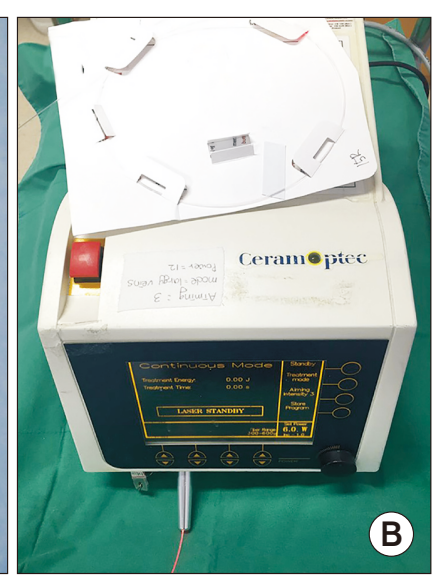

(B)
Fig. 1. The instruments used for officebased laser surgery. (A) Transnasal esophagoscope, $60 \mathrm{~cm}$ length with 5.5 mm diameter, 1 working channel, (B) diode laser instruments. 
koplakia and laryngeal hair, the lesion showed significant improvement, yet required repeated procedures (Fig. 3). On the other hand, patients with contact granuloma, sub-

Table 1. Patient data and procedure outcomes

\begin{tabular}{rrlcl}
\hline No. & Sex/Age & \multicolumn{1}{c}{ Diagnosis } & $\begin{array}{c}\text { Number of } \\
\text { procedure }\end{array}$ & \multicolumn{1}{c}{ Results } \\
\hline 1 & $\mathrm{M} / 73$ & VF polyp & 1 & NED \\
2 & $\mathrm{M} / 41$ & VF polyp & 1 & NED \\
3 & $\mathrm{~F} / 43$ & VF polyp & 1 & NED \\
4 & $\mathrm{M} / 46$ & Contact granuloma & 1 & Partial \\
5 & $\mathrm{~F} / 66$ & Leukoplakia & 1 & Marked \\
6 & $\mathrm{~F} / 57$ & Subglottic stenosis & 3 & Partial \\
7 & $\mathrm{M} / 75$ & Laryngeal hair & 6 & Marked \\
8 & $\mathrm{M} / 23$ & Papillomatosis & 1 & Recur $\rightarrow \mathrm{G} / \mathrm{A}$ \\
9 & $\mathrm{M} / 57$ & Papillomatosis & 2 & NED \\
10 & $\mathrm{M} / 69$ & Epiglottic tumor & 1 & NED \\
11 & $\mathrm{M} / 38$ & Intubation granuloma & 1 & Partial \\
12 & $\mathrm{M} / 59$ & Tracheal stenosis & 1 & Partial \\
\hline
\end{tabular}

VF, vocal fold; NED, no evidence of disease; Partial, partial remission; Marked, markedly decreased; G/A, general anesthesia glottic stenosis and tracheal lesion showed partial remission with the laser surgery. Only one patient required conversion to general anesthesia after office-laser surgery; this patient had laryngeal papillomatosis with invisible surface and inappropriate exposure, making it difficult for fiberoptic endoscope to reach the lesion.

\section{DISCUSSION}

Our study demonstrated the feasibility of in-office treatment of laryngeal disease by fiberoptic endoscope guided laser. Neither immediate nor long-term complication were found during the study. Some cases required repeated procedures, but this is only the innate characteristics of the disease itself, not the weakness of the procedure. Laryngeal papilloma, granuloma, and airway stenosis are the most frequently recurred disease among benign laryngeal lesion, and this is relevant in laryngeal microsurgery under general anesthesia. We experienced 1 case with laryngeal papilloma where surgical plan changed after failure of fiberoptic endoscope guided laser
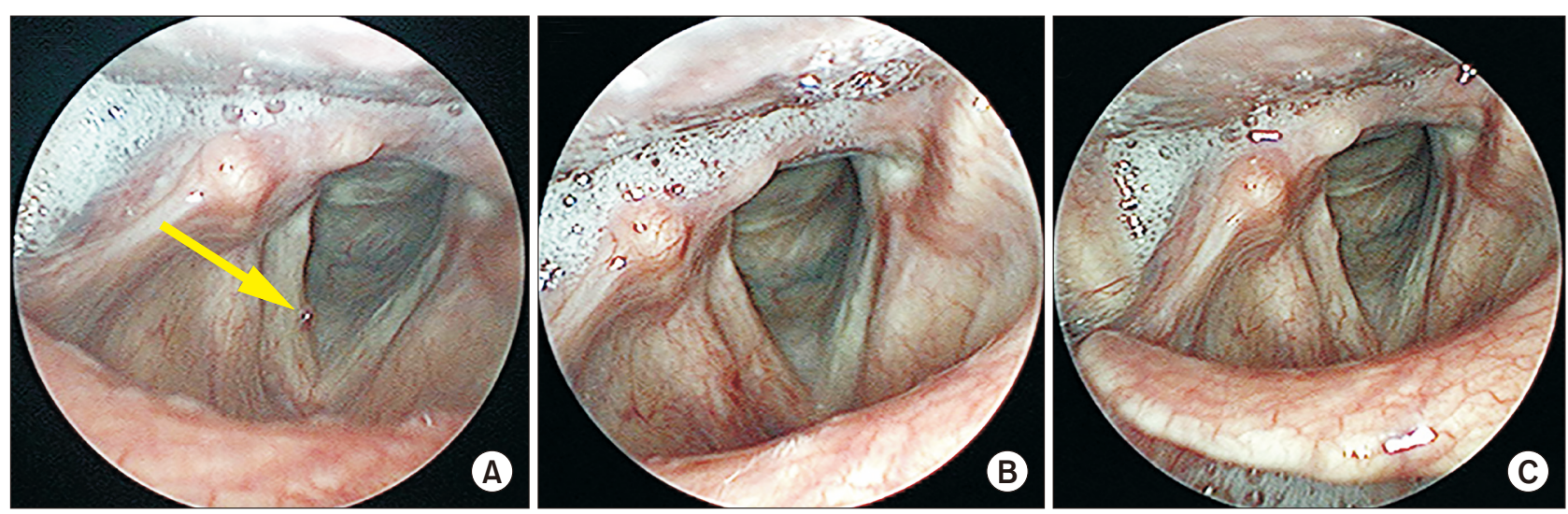

Fig. 2. Forty one year old male with vocal fold polyp. (A) Preoperative, (B) Postoperative 1 month, (C) Postoperative 3 months.
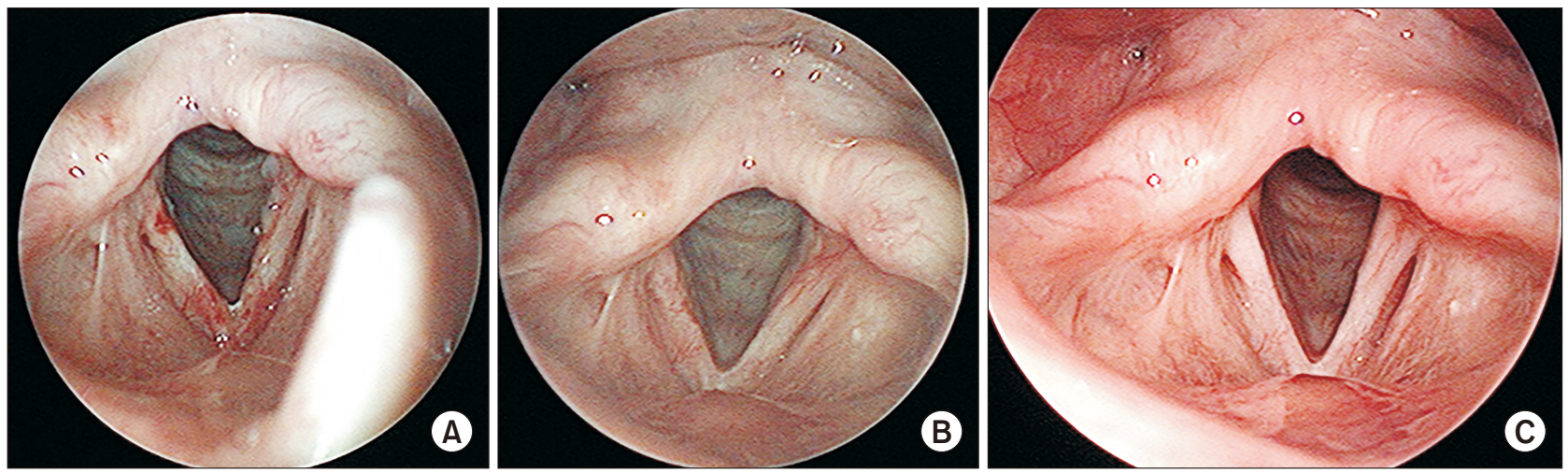

Fig. 3. Fifty seven year old male with papillomatosis. (A) Preoperative, (B) Postoperative 1 month, (C) Postoperative 3 months. 
surgery. Therefore, we believe that surgeon's experience for patient selection is by far the most important factor for treatment success.

Since its introduction in field of laryngology over 30 years ago, lasers have facilitated important innovations that have now evolved into office-based surgery. ${ }^{6}$ These advancements accommodated well to our specialty, leading surgeons to minimally invasive surgical approaches since mirror-guided interventions in the nineteenth century. ${ }^{7}$ Initially, patients with benign vocal fold lesion, such as polyps, was the only candidate for office based laser surgery. However, candidate for in-office laser surgery of laryngeal disease is expanding due to the improvement of instruments, such as endoscope monitoring system and laser system. In numerous medical centers, fiber-based technologies have already caused many procedures to be performed by means of local anesthesia in the clinic or office, especially for patients with chronic diseases such as papillomatosis and dysplasia. This approach is likely to expand significantly due to diminished patient morbidity of the procedure, along with socioeconomic pressures of healthcare delivery.

Indeed, office based laser surgery for laryngeal disease have proven to be better than conventional treatment in several subjective parameters evaluated by patients. Rees et al demonstrated that in a retrospective survey of 89 subjects who underwent pulse-dye laser in the office, 87 percent of the patients stated that they preferred office-based laryngeal laser surgery over operating room surgeries; fifty-four of these subjects had previous operating room surgeries for the same pathology. ${ }^{3}$ Eightythree percent of the patients demonstrated that they had less discomfort with office-based laryngeal laser surgery, compared to that of operating room surgeries. Office based laryngeal laser surgery is less costly and more expedient for patients, especially for those with laryngeal and pharyngeal pathology that requires multiple procedures as recurrent respiratory papillomatosis and esophageal stricture. The major advantage of office-based laryngeal surgery is needlessness of general anesthesia for patients with systemic disease, such as cardiovascular disease. ${ }^{8}$ High resolution, distal chip, and video laryngoscopes enable surgeons to detect disease earlier than before, and hemostatic cutting of laser improves safety of local procedure by preventing aspiration. Both the surgeon's and patient's needs to accept surgery under general anesthesia are reduced as the procedure can be performed under local anesthesia at the time of the patient's routine visits. ${ }^{9.10}$ Such change enables patients to maintain their lifestyle, and protects them from possible risks from general anesthesia.

Despite the aforementioned advantages, office based laser surgery has a number of limitations. First of all, office-based laser surgery cannot achieve the pathologic specimen in cases of small lesions when compared to that of traditional microsurgery. This may contraindicate several conditions where the accurate diagnosis is needed for differentiating the laryngeal malignancy. Second of all, in cases of invisible surface under vertical direction, approach to pathology can be difficult. As mentioned earlier, patient selection is one of the most important factors for the success of approach, and we believe that experience is needed for proper selection. Lastly, proper resection margin cannot be achieved through the vaporization technique. Therefore, recurrent benign diseases, including papillomatosis or dysplastic lesions, may be contraindicated in some cases.

We want to emphasize that precise targeting is difficult due to patient's coughing and swallowing under shallow anesthesia. Therefore, deep anesthesia of entire laryngeal mucosa is very important for successful and accurate procedure.

The role of both lasers and office-based laryngeal surgeries will continue to evolve. The high-resolution, distalchip-camera endoscopic technology, various laser types and local anesthetics make office-based laryngeal surgery highly appealing in many ways. One of the weakness of these clinical advancements is the cost required to install the laser technology in institutions and surgeons' offices. Furthermore, critical development of these new lasers is limited by the relatively small numbers of patients with the laryngeal disorders, which discourages industry from investing substantial research and development funding. To solve this problem, our hope is that laryngology will continue to serve as a model for high-performance minimally invasive surgery that can be translated to other mucosal diseases of the upper and lower aerodigestive tract, genito-urinary organs and the cervix. Broader use of these new lasers in other surgical disciplines should diminish costs for all surgeons and their associated institutions.

\section{ACKNOWLEDGMENTS}

This research was supported by the Clinical Trial Center of Korea University Anam Hospital (11500931), the Korea Health Technology R\&D Project (HI14C0748) through the Korea Health Industry Development Institute (KHIDI) by the Ministry of Health \& Welfare. 


\section{REFERENCES}

1. Sridharan S, Achlatis S, Ruiz R, Jeswani S, Fang Y, Branski RC, et al. Patient-based outcomes of in-office KTP ablation of vocal fold polyps. Laryngoscope 2014;124:1176-9.

2. Zeitels SM, Burns JA. Office-based laryngeal laser surgery with local anesthesia. Curr Opin Otolaryngol Head Neck Surg 2007;15:141-7.

3. Rees CJ, Halum SL, Wijewickrama RC, Koufman JA, Postma GN. Patient tolerance of in-office pulsed dye laser treatments to the upper aerodigestive tract. Otolaryngol Head Neck Surg 2006;134:1023-7.

4. McMillan K, Shapshay SM, McGilligan JA, Wang Z, Rebeiz EE. A 585-nanometer pulsed dye laser treatment of laryngeal papillomas: preliminary report. Laryngoscope 1998;108:968-72.

5. Valdez TA, McMillan K, Shapshay SM. A new laser treatment for vocal cord papilloma--585-nm pulsed dye. Otolaryngol Head Neck Surg 2001;124:421-5.

6. Zeitels SM, Burns JA. Laser applications in laryngology: past, present, and future. Otolaryngol Clin North Am 2006;39:159-72.

7. Zeitels SM, Burns JA, Akst LM, Hillman RE, Broadhurst MS, Anderson RR. Office-based and microlaryngeal applications of a fiber-based thulium laser. Ann Otol Rhinol Laryngol 2006;115:891-6.

8. Centric A, Hu A, Heman-Ackah YD, Divi V, Sataloff RT. Officebased pulsed-dye laser surgery for laryngeal lesions: a retrospective review. J Voice 2014;28:262.e9-262.e12.

9. Shah MD, Johns MM 3rd. Office-based laryngeal procedures. Otolaryngol Clin North Am 2013;46:75-84.

10. Woo P. Office-based laryngeal procedures. Otolaryngol Clin North Am 2006;39:111-33. 